\title{
Estimates for the ruin probability in the classical risk model with constant interest force in the presence of heavy tails ${ }^{\text {is }}$
}

\author{
Dimitrios Konstantinides $^{\mathrm{a}}$, Qihe Tang ${ }^{\mathrm{b}, *}$, Gurami Tsitsiashvili ${ }^{\mathrm{c}}$ \\ a Department of Statistics and Actuarial Science, University of the Aegean, Samos 83200, Greece \\ ${ }^{\mathrm{b}}$ Department of Quantitative Economics, University of Amsterdam, Roetersstraat 11, 1018 WB Amsterdam, The Netherlands \\ c Institute of Applied Mathematics, FEB RAS, Radio Str. 7, Vladivostok 690041, Russia
}

Received July 2001; received in revised form April 2002; accepted 26 September 2002

\begin{abstract}
In this paper we investigate the ruin probability in the classical risk model under a positive constant interest force. We restrict ourselves to the case where the claim size is heavy-tailed, i.e. the equilibrium distribution function (e.d.f.) of the claim size belongs to a wide subclass of the subexponential distributions. Two-sided estimates for the ruin probability are developed by reduction from the classical model without interest force.
\end{abstract}

() 2002 Elsevier Science B.V. All rights reserved.

Keywords: Classical risk model; Constant interest force; The ruin probability; Subexponential distribution

\section{Introduction}

The classical risk model with homogeneous Poisson arrival process, constant premium rate and constant interest force has been investigated by many authors such as Sundt and Teugels (1995, 1997), Asmussen (1998), Klüppelberg and Stadtmüller (1998) and Kalashnikov and Konstantinides (2000). We address in the present paper two-sided bounds for the ruin probability in this model. The well-known inequalities in the classical risk model without interest force enable us to derive accurate two-sided estimates. The idea of the reduction is not new, but only recently this method became effective as the necessary tools were accumulated. This approach is also applicable to the study of the convergence rate of the ruin probability approximations.

We suppose that the claim sizes, $\left(Z_{k}\right)_{k \geq 1}$, form a sequence of i.i.d. non-negative r.v.'s, with a common d.f. $B(x)=1-\bar{B}(x)=P\left(Z_{1} \leq x\right), x \geq 0$, and a finite expectation $b$. Throughout this paper, the d.f. $B$ always satisfies $\bar{B}(x)>0$ for all $x \geq 0$. We denote by

$$
F(x)=\frac{1}{b} \int_{0}^{x} \bar{B}(z) \mathrm{d} z, \quad x \geq 0
$$

the equilibrium distribution function (e.d.f.) of the d.f. $B$. We assume that, as usual, the claim arrival times constitute a homogeneous Poisson process $(N(t))_{t \geq 0}$, which is independent of $\left(Z_{k}\right)_{k \geq 1}$ and has an intensity $\lambda>0$. Therefore,

\footnotetext{
th This work was supported by Dutch Organization for Scientific Research (project No. NWO 42511013) and NATO research program 2001.

* Corresponding author. Tel.: +31-20-5254107; fax: +31-20-5254349.

E-mail addresses: konstant@ aegean.gr (D. Konstantinides), q.tang@uva.nl (Q. Tang), guram@iam-mail.febras.ru (G. Tsitsiashvili).
} 
the compound Poisson process $X(t)=\sum_{k=1}^{N(t)} Z_{k}$ represents the total claim amount accumulated up to time $t \geq 0$, with $X(t)=0$ when $N(t)=0$. We write $\rho=\lambda b$ and let $c$ be the constant gross premium rate which is not necessarily positive. We assume that there exists a constant interest force $r>0$ which affects the risk process. Let $u>0$ be the initial surplus of the insurance company, then the total surplus up to time $t$, represented by $U_{r}(t)$, satisfies the equation

$$
U_{r}(t)=u \mathrm{e}^{r t}+c \int_{0}^{t} \mathrm{e}^{r z} \mathrm{~d} z-\int_{0}^{t} \mathrm{e}^{r(t-z)} \mathrm{d} X(z), \quad t \geq 0 .
$$

The ultimate ruin probability for this risk process is then defined by

$$
\psi_{r}(u)=P\left(\inf _{t \geq 0} U_{r}(t)<0 \mid U_{r}(0)=u\right), \quad u \geq 0 .
$$

As many recent references in the fields of risk theory, we are interested in heavy-tailed claim sizes. The most important class of heavy-tailed d.f.'s is the subexponential class $\mathcal{S}$. By definition, a d.f. $F$ supported on $[0, \infty)$ belongs to the class $\mathcal{S}$ iff for any (or equivalently for some) $n \geq 2$, it holds that

$$
\lim _{x \rightarrow \infty} \frac{\overline{F^{* n}}(x)}{\bar{F}(x)}=n
$$

where $F^{* n}$ denotes the $n$-fold convolution of the d.f. $F$. We refer to Embrechts et al. (1997), Rolski et al. (1999) and Asmussen (2000) for thorough reviews of the applications of the class $\mathcal{S}$ to insurance and finance.

It is well known that, if the e.d.f. $F$ of the claim size belongs to the class $\mathcal{S}$ and the safety loading condition $\rho<c$ holds, then

$$
\psi_{0}(u) \sim \frac{\rho}{c-\rho} \bar{F}(u), \quad u \rightarrow \infty,
$$

see Feller (1971) and Embrechts and Veraverbeke (1982). Based on the classical asymptotics (1.2), Kalashnikov and Tsitsiashvili $(1999,2000)$ and Mikosch and Nagaev (2001) introduced as an auxiliary function the relative error of the approximation (1.2)

$$
\Delta(u)=\frac{\psi_{0}(u)}{(\rho /(c-\rho) \bar{F}(u)}-1, \quad u \geq 0 .
$$

Then they studied the bounds and the convergence rate of the quantity $\Delta(u) \rightarrow 0$ as $u \rightarrow \infty$.

Recently, Asmussen (1998), Klüppelberg and Stadtmüller (1998) and Kalashnikov and Konstantinides (2000) considered the ruin probability $\psi_{r}(u)$ with the constant interest force $r>0$. They established an asymptotic formula which is similar to the classical asymptotics (1.2).

In this paper we continue the work of the cited papers. Asmussen's formula for the ruin probability $\psi_{r}(u)$ is the starting point of our present investigation. First, in Section 2, after some analysis on Asmussen's formula, we introduce an additional condition on the claim size distribution. By this condition we characterize a new broad subexponential subclass. Then, based on Asmussen's formula we drive some two-sided bounds for the ruin probability $\psi_{r}(u)$ in Section 3. Some examples are laid in Section 4, and numerical results are in Section 5.

\section{Main results}

\subsection{On an asymptotic formula for the ruin probability}

Recall that $F$ represents the e.d.f. of the claim size distribution $B$. Under the assumption $F \in \mathcal{S}$, Asmussen (1998) first established an asymptotic formula for the ruin probability $\psi_{r}(u)$ with $r>0$ that 


$$
\psi_{r}(u) \sim \frac{\lambda}{r} \int_{u}^{\infty} \bar{B}(z) \frac{\mathrm{d} z}{z}, \quad u \rightarrow \infty .
$$

Almost simultaneously, Klüppelberg and Stadtmüller (1998) used sophisticated analytical arguments to derive (2.1) in the presence of claim size having regularly varying tail with exponent $\alpha>1$, i.e.

$$
\bar{B}(x)=x^{-\alpha} L(x), \quad x \geq 0,
$$

where $L(x)$ is a positive function which is slowly varying as $x$ tends to infinity (we rewrite $B \in \mathcal{R}_{-\alpha}$ as usual). Later, Kalashnikov and Konstantinides (2000) provided a simple proof for Asmussen's formula (2.1) for the whole subexponential case. In the proofs provided by Asmussen (1998) and Kalashnikov and Konstantinides (2000), however, some supporting arguments should be required. This was pointed out by Asmussen et al. (2002, pp. 403 and 404). We also refer readers to the proof of Lemma 3 in Kalashnikov and Konstantinides (2000), where the authors used an implication that, for infinitesimal quantities $A_{i}(u), 1 \leq i \leq 4$, if $A_{1}(u) \sim A_{2}(u)$ and $A_{3}(u) \sim A_{4}(u)$ then

$$
A_{1}(u)-A_{3}(u) \sim A_{2}(u)-A_{4}(u)
$$

which is wrong, in general.

We prove that (2.1) remains valid under an additional restriction. That is we have the following theorem.

Theorem 2.1. In the classical risk model with a constant interest force $r>0$, the asymptotics (2.1) is true if the e.d.f. $F \in \mathcal{S}$ and that, for some $v>1$,

$$
\limsup _{x \rightarrow \infty} \frac{\bar{F}(v x)}{\bar{F}(x)}<1 \text {. }
$$

Clearly, if the claim size distribution $B \in \mathcal{R}_{-\alpha}$ for some $\alpha>1$ then the e.d.f. $F$ satisfies all the conditions asked in Theorem 2.1.

Motivated by Theorem 2.1 we introduce a new subclass of subexponential d.f.'s below.

Definition 2.1. Let $F$ be a d.f. supported on $[0, \infty)$. We say that $F$ belongs to $\mathcal{A}$ if $F \in \mathcal{S}$ and (2.2) holds for some $v>1$.

We point out that the class $\mathcal{A}$ covers almost all the well-known subexponential d.f.'s. In fact, by its definition one easily checks the following remark.

Remark 2.1. All the d.f.'s listed in Table 1.2.6 in Embrechts et al. (1997) belong to the class $\mathcal{A}$. Namely the Pareto, the log-normal, the Weibull, the log-gamma, the Burr, the Benktander I and II distributions are included in the class $\mathcal{A}$.

With the notation

$$
D(x)=1-\frac{x}{\bar{F}(x)} \int_{x}^{\infty} \bar{F}(z) \frac{\mathrm{d} z}{z^{2}}, \quad x \geq 0,
$$

we immediately obtain from Theorem 2.1 the following corollary.

Corollary 2.1. In the classical risk model with a constant interest force $r>0$, the asymptotic formula

$$
\psi_{r}(u) \sim \frac{\rho}{r u} D(u) \bar{F}(u), \quad u \rightarrow \infty,
$$

is true if the e.d.f. $F \in \mathcal{A}$. 
Proof. From (2.1) we have

$$
\psi_{r}(u) \sim \frac{\lambda}{r} \int_{u}^{\infty} \bar{B}(z) \frac{\mathrm{d} z}{z}=\frac{\lambda b}{r}\left[-\int_{u}^{\infty} \frac{1}{z} \mathrm{~d} \bar{F}(z)\right],
$$

the right-hand side of which equals to $(\rho / r u) D(u) \bar{F}(u)$ by integration by parts.

\subsection{Two-sided estimates for the ruin probability}

In order to investigate two-sided estimates for the ruin probability $\psi_{r}(u)$ with $r>0$, now we seek for another auxiliary function which plays a similar role in the present situation as that of $\Delta(u)$ in the case without interest force. The asymptotic formula (2.3) urges us to use

$$
\Gamma(u)=\frac{\psi_{r}(u)}{(\rho / r u) D(u) \bar{F}(u)}-1, \quad u \geq 0 .
$$

Obviously, $\Gamma(u)$ represents the relative error of the approximation (2.3).

Now we state the main result of the paper.

Theorem 2.2. In the classical risk model, if $\rho<c$, then for any $u>0$ we have that

$$
\Gamma_{-}(u) \leq \Gamma(u) \leq \Gamma_{+}(u),
$$

where

$$
\begin{aligned}
& \Gamma_{-}(u)=-\frac{1}{D(u)}\left(\frac{c}{c+r u}+\frac{\bar{\Delta}(u)+\psi_{0}(u)}{1-\psi_{0}(u)}[1-D(u)]\right), \\
& \Gamma_{+}(u)=-\frac{c}{c+r u}+\frac{1}{D(u)}\left(\frac{c}{c+r u}+\frac{\Delta(u)+\psi_{0}(u)}{1-\psi_{0}(u)}\right),
\end{aligned}
$$

and $\bar{\Delta}(u)=\sup _{x \geq u} \Delta(x)$.

The following bound for $|\Gamma(u)|$ is sometimes more convenient for applications.

Corollary 2.2. Under the conditions of Theorem 2.2, we have that, for any $u>0$,

$$
|\Gamma(u)| \leq \frac{1}{D(u)}\left(\frac{c}{c+r u}+\frac{\bar{\Delta}(u)+\psi_{0}(u)}{1-\psi_{0}(u)}\right) .
$$

Proof. The proof is straightforward from (2.4).

Note that in Theorem 2.2 and Corollary 2.2, we did not require any condition on the tail behavior of the claim size. Now we assume that (2.2) holds for some $v>1$. By Lemma 3.3 we know that (2.2) holds for any $v>1$. Thus, for any $v>1$, there exists some $l(v)=l_{F}(v)>0$ such that

$$
d(v)=\sup _{x>l(v)} \frac{\bar{F}(v x)}{\bar{F}(x)}<1 .
$$

For convenience we write $d(1)=1$. With the $v, l(v)$ and $d(v)$ given above, we introduce

$$
d(v, k)=\sup _{x>l(v)} \frac{\bar{F}\left(v^{k} x\right)}{\bar{F}(x)}, \quad \sigma(v)=\sum_{k=1}^{\infty} \frac{d(v, k-1)-d(v, k)}{v^{k}} .
$$

Obviously, it holds for any $v>1$ that $d(v, 0)=d(1)=1$ and $d(v, 1)=d(v)$. We obtain the following theorem. 
Theorem 2.3. In addition to the conditions of Theorem 2.2, we assume that (2.2) holds for some $v>1$. Then the following inequalities hold for any $v>1$ and $u>l(v)$ :

$$
\begin{aligned}
\Gamma_{-}(u) & \geq-\frac{1}{\sigma(v)}\left(\frac{c}{c+r u}+\frac{\bar{\Delta}(u)+(\rho /(c-\rho)) \bar{F}(u)[1+\Delta(u)]}{1-(\rho /(c-\rho)) \bar{F}(u)[1+\Delta(u)]}[1-\sigma(v)]\right), \\
\Gamma_{+}(u) & \leq-\frac{c}{c+r u}+\frac{1}{\sigma(v)}\left(\frac{c}{c+r u}+\frac{\Delta(u)+(\rho /(c-\rho)) \bar{F}(u)[1+\Delta(u)]}{1-(\rho /(c-\rho)) \bar{F}(u)[1+\Delta(u)]}\right), \\
|\Gamma(u)| & \leq \frac{1}{\sigma(v)}\left(\frac{c}{c+r u}+\frac{\bar{\Delta}(u)+(\rho /(c-\rho)) \bar{F}(u)[1+\Delta(u)]}{1-(\rho /(c-\rho)) \bar{F}(u)[1+\Delta(u)]}\right) .
\end{aligned}
$$

We notice that all the bounds given in Theorem 2.3 essentially depends on three quantities $c /(c+r u), \Delta(u)$ and $\bar{F}(u)$. This enables us to investigate the convergence rate of the deviation $\Gamma(u)$ to 0 as $u \rightarrow \infty$ by reduction from the existing results in the literature. We refer readers to Kalashnikov and Tsitsiashvili (2000) for some estimates for $\Delta(u)$ or $\psi_{0}(u)$, and to Mikosch and Nagaev (2001) for some details on the convergence rate of $\Delta(u)$ to 0 as $u \rightarrow \infty$.

\section{Proofs of the main results}

\subsection{Some lemmas}

In this section we propose some lemmas about the class $\mathcal{A}$. They will play crucial roles in the proofs of our main results.

Lemma 3.1. Let $F$ be a d.f. supported on $[0, \infty)$. If (2.2) holds for some $v>1$, then, for all $x \geq l(v)$,

$$
D(x) \geq \sigma(v) \geq \frac{1-d(v)}{v}>0,
$$

where the notations involved were given in Section 2.

Proof. Recall the definitions of $d(v), d(v, k)$ and $\sigma(v)$. We have that the quantity $d(v, k)$ is non-decreasing in $k \geq 0$. Therefore,

$$
\sigma(v) \geq \frac{d(v, 0)-d(v, 1)}{v}=\frac{1-d(v)}{v}>0, \quad v>1 .
$$

As for the first inequality in (3.1), we have

$$
D(x) \geq 1-\frac{x}{\bar{F}(x)} \sum_{k=1}^{\infty} \bar{F}\left(v^{k-1} x\right) \int_{v^{k-1} x}^{v^{k} x} \frac{\mathrm{d} z}{z^{2}} \geq 1-\sum_{k=1}^{\infty} d\left(v^{k-1}\right)\left(\frac{1}{v^{k-1}}-\frac{1}{v^{k}}\right)=\sigma(v) .
$$

This ends the proof.

Lemma 3.2. Let $F$ be a d.f. supported on $[0, \infty)$. Then

$$
A_{F}=\limsup _{x \rightarrow \infty} \frac{x}{\bar{F}(x)} \int_{x}^{\infty} \bar{F}(z) \frac{\mathrm{d} z}{z^{2}}<1
$$

if and only if (2.2) holds for some $v>1$. 
Proof. The proof of the "if" assertion follows from Lemma 3.1. Now we only need to prove the "only if" assertion. Clearly, for any $v>1$,

$$
\frac{x}{\bar{F}(x)} \int_{x}^{\infty} \bar{F}(z) \frac{\mathrm{d} z}{z^{2}} \geq \frac{x}{\bar{F}(x)} \int_{x}^{v x} \bar{F}(z) \frac{\mathrm{d} z}{z^{2}} \geq \frac{x}{\bar{F}(x)} \bar{F}(v x)\left(\frac{1}{x}-\frac{1}{v x}\right) .
$$

It follows that:

$$
\limsup _{x \rightarrow \infty} \frac{\bar{F}(v x)}{\bar{F}(x)} \leq \frac{v}{v-1} \limsup _{x \rightarrow \infty} \frac{x}{\bar{F}(x)} \int_{x}^{\infty} \bar{F}(z) \frac{\mathrm{d} z}{z^{2}}=\frac{v}{v-1} A_{F}, \quad v>1 .
$$

So (2.2) holds for all $v>\left(1-A_{F}\right)^{-1}>1$. This ends the proof.

Lemma 3.3. Let $F$ be a d.f. supported on $[0, \infty)$ with a density function $f(x)$ which is eventually non-increasing. Then the following statements are equivalent:

( $\left.I_{1}\right)$ (2.2) holds for some $v>1$;

(I $)$ (2.2) holds for any $v>1$;

$\left(I_{3}\right)$ the hazard rate of $F, q(x)=f(x) / \bar{F}(x)$, satisfies

$$
\liminf _{x \rightarrow \infty} x q(x)>0 \text {. }
$$

Proof. We prove the lemma by the following order of implications: $I_{1} \Rightarrow I_{3} \Rightarrow I_{2} \Rightarrow I_{1}$.

(1) $I_{1} \Rightarrow I_{3}$. For the fixed $v>1$ in $I_{1}$ and all sufficiently large $x>0$,

$$
\frac{\bar{F}(v x)}{\bar{F}(x)}=1-\frac{\int_{x}^{v x} f(t) \mathrm{d} t}{\bar{F}(x)} \geq 1-(v-1) x q(x),
$$

from which it follows that:

$$
\liminf _{x \rightarrow \infty} x q(x) \geq \frac{1}{v-1} \liminf _{x \rightarrow \infty}\left(1-\frac{\bar{F}(v x)}{\bar{F}(x)}\right)>0 .
$$

(2) $I_{3} \Rightarrow I_{2}$. For any fixed $v>1$ and all large $x>0$,

$$
\frac{\bar{F}(v x)}{\bar{F}(x)}=\frac{\bar{F}(v x)}{\int_{x}^{v x} f(t) \mathrm{d} t+\bar{F}(v x)} \leq \frac{\bar{F}(v x)}{(v-1) x f(v x)+\bar{F}(v x)}=\frac{1}{(v-1) x q(v x)+1},
$$

which, together with (3.4), implies that (2.2) holds for any $v>1$.

(3) $I_{2} \Rightarrow I_{1}$. This step is trivial.

Remark 3.1. Let $F$ be the e.d.f. of the claim size distribution $B$. We write

$$
L_{1}(v)=\liminf _{x \rightarrow \infty} \frac{\bar{F}(v x)}{\bar{F}(x)} \quad \text { and } \quad L_{2}(v)=\limsup _{x \rightarrow \infty} \frac{\bar{F}(v x)}{\bar{F}(x)}, \quad v>1 .
$$

Clearly, Lemma 3.3 indicates that if $L_{2}(v)=1$ for some $v>1$ then it holds for all $v>1$. Furthermore, going along the line of the proof of Lemma 3.3 we also obtain that

$$
L_{1}(v)<1 \quad \exists v>1 \Longleftrightarrow L_{1}(v)<1 \quad \forall v>1 \Longleftrightarrow \limsup _{x \rightarrow \infty} x q(x)>0 .
$$

So we have that if $L_{1}(v)=1$ for some $v>1$ then it holds for all $v>1$. From these discussions we can classify all 
possibilities of the values of $L_{1}(v)$ and $L_{2}(v)$ into three cases:

1. $L_{1}(v)=L_{2}(v)=1 \forall v>1$;

2. $L_{1}(v)<1$ but $L_{2}(v)=1 \quad \forall v>1$;

3. $L_{2}(v)<1 \quad \forall v>1$.

The first case indicates that $\bar{F}(x)$ is slowly varying as $x \rightarrow \infty$. The third case is just the fundamental assumption of the present paper. Our past experience shows that problems for the second case are often very complicated.

\subsection{Proof of Theorem 2.1}

We shall need two auxiliary functions

$$
G_{r}(u)=1-\frac{\psi_{r}(u)}{\psi_{r}(0)}, \quad k_{r}(u)=\int_{u}^{\infty} z \mathrm{~d} G_{r}(z), \quad u \geq 0,
$$

and a notation

$$
K_{r}=\rho \frac{1-\psi_{r}(0)}{\psi_{r}(0)},
$$

which were first introduced by Sundt and Teugels (1995). These expressions enable us to take the following representation for the ruin probability:

$$
\psi_{r}(u)=\frac{\rho}{K_{r}+\rho}\left(\frac{k_{r}(u)}{u}-\int_{u}^{\infty} k_{r}(z) \frac{\mathrm{d} z}{z^{2}}\right), \quad u \geq 0 .
$$

Further we shall use the following two-sided bounds of $k_{r}(u)$ :

$$
\frac{\left(\rho+K_{r}\right) u}{c+r u} \bar{F}(u) \leq k_{r}(u) \leq \frac{\left(\rho+K_{r}\right)(c-\rho)}{r \rho} \frac{\psi_{0}(u)}{1-\psi_{0}(u)},
$$

see Kalashnikov and Konstantinides (2000).

We assume temporarily that the safety loading condition $\rho<c$ holds. Therefore the classical formula (1.2) is valid. The inequalities (3.6), together with the asymptotics (1.2), give the relationship

$$
k_{r}(u) \sim \frac{\rho+K_{r}}{r} \bar{F}(u), \quad u \rightarrow \infty .
$$

Hence,

$$
\limsup _{u \rightarrow \infty} \frac{\int_{u}^{\infty} k_{r}(z)\left(\mathrm{d} z / z^{2}\right)}{k_{r}(u) / u}=\limsup _{u \rightarrow \infty} \frac{u}{\bar{F}(u)} \int_{u}^{\infty} \bar{F}(z) \frac{\mathrm{d} z}{z^{2}} .
$$

It is easy to see that, if (3.3) holds then we are allowed to substitute (3.7) into (3.5) on the way to the asymptotic relationship (1.2)

$$
\psi_{r}(u) \sim \frac{\rho}{K_{r}+\rho} \frac{\rho+K_{r}}{r}\left(\frac{1}{u} \bar{F}(u)-\int_{u}^{\infty} \bar{F}(z) \frac{\mathrm{d} z}{z^{2}}\right)=\frac{\lambda}{r} \int_{u}^{\infty} \bar{B}(z) \frac{\mathrm{d} z}{z}, \quad u \rightarrow \infty .
$$

But we have proved in Lemma 3.2 that (3.3) is equivalent to the assertion that (2.2) holds for some $v>1$, which is implied by the membership of $F$ in $\mathcal{A}$. This proves Theorem 2.1 for the case where $\rho<c$.

If $\rho \geq c$, then, by the same argument as the proof of Lemma 4 in Kalashnikov and Konstantinides (2000), we can still obtain (2.1). Hence, the validity of (2.1) is independent of the safety loading condition. This ends the proof. 


\subsection{Proof of Theorem 2.2}

From (3.5) and (3.6) and noting that the functions $\bar{\Delta}(z)$ and $\psi_{0}(z)$ are non-increasing in $z \geq 0$, we derive the following lower bound of $\psi_{r}(u)$ :

$$
\begin{aligned}
\psi_{r}(u) & \geq \frac{\rho}{c+r u} \bar{F}(u)-\frac{c-\rho}{r} \int_{u}^{\infty} \frac{\psi_{0}(z)}{1-\psi_{0}(z)} \frac{\mathrm{d} z}{z^{2}} \\
& \geq \frac{\rho}{r u} \bar{F}(u)\left(\frac{r u}{c+r u}-\frac{u}{\left[1-\psi_{0}(u)\right] \bar{F}(u)} \int_{u}^{\infty}[1+\Delta(z)] \bar{F}(z) \frac{\mathrm{d} z}{z^{2}}\right) \\
& \geq \frac{\rho \bar{F}(u)}{r u}\left(\frac{r u}{c+r u}-\frac{1+\bar{\Delta}(u)}{1-\psi_{0}(u)}[1-D(u)]\right) \\
& =\frac{\rho}{r u} \bar{F}(u) D(u)\left[1-\frac{1}{D(u)}\left(\frac{c}{c+r u}+\frac{\bar{\Delta}(u)+\psi_{0}(u)}{1-\psi_{0}(u)}[1-D(u)]\right)\right] .
\end{aligned}
$$

Similarly, we obtain the upper bound of $\psi_{r}(u)$ as follows:

$$
\begin{aligned}
\psi_{r}(u) & \leq \frac{c-\rho}{r u} \frac{\psi_{0}(u)}{1-\psi_{0}(u)}-\rho \int_{u}^{\infty} \bar{F}(z) \frac{\mathrm{d} z}{z(c+r z)} \\
& \leq \frac{\rho}{r u} \bar{F}(u)\left(\frac{\psi_{0}(u)}{(\rho /(c-\rho)) \bar{F}(u)\left[1-\psi_{0}(u)\right]}-\frac{r u}{c+r u}[1-D(u)]\right) \\
& =\frac{\rho}{r u} \bar{F}(u) D(u)\left(\frac{r u}{c+r u}+\frac{1}{D(u)}\left[\frac{c}{c+r u}+\frac{\Delta(u)+\psi_{0}(u)}{1-\psi_{0}(u)}\right]\right) .
\end{aligned}
$$

By these two bounds in (3.8) and (3.9), we get the proof of (2.4).

\subsection{Proof of Theorem 2.3}

Note that, for our case, $F$ has a density function $f(x)=b^{-1} \bar{B}(x)$ which is non-increasing in $x \geq 0$ and tends to 0 as $x \rightarrow \infty$. Since (2.2) holds for some $v>1$, then by Lemmas 3.1 and 3.3, for any $v>1$, there exists some $l(v)>0$ such that (3.1) holds for all $u>l(v)$. The remaining proof of Theorem 2.3 is trivial.

\section{Examples}

Example 4.1. Now we put forward some concrete examples to illustrate how to determine the values of $l(v), d(v)$, $\sigma(v)$, and $D(u)$ involved in our main results:

(1) Let $F$ be the Pareto distribution with $a>1$ and $\kappa>0$, i.e. the tail of $F$ satisfies

$$
\bar{F}(x)= \begin{cases}1 & \text { when } x \leq \kappa, \\ \left(\frac{\kappa}{x}\right)^{a} & \text { when } x>\kappa .\end{cases}
$$

Clearly, for any $v>1$, we have

$$
d(v)=v^{-a}, \quad \sigma(v)=\frac{v^{a}-1}{v^{a+1}-1} \quad \text { and } \quad l(v)=\kappa .
$$


(2) Let $F$ be the Burr distribution with tail

$$
\bar{F}(x)=\left(\frac{\kappa}{\kappa+x^{s}}\right)^{a}, \quad a, \kappa, s>0, \quad x \geq 0 .
$$

Modeling the proof of (3.2) with slight adjustment yields that, for any $v>1$ and $u>0$,

$$
D(u) \geq \frac{1}{v}\left[1-\frac{\bar{F}(v u)}{\bar{F}(u)}\right]=\frac{1}{v}\left[1-\left(\frac{\kappa+u^{s}}{\kappa+(v u)^{s}}\right)^{a}\right] \sim \frac{v^{s a}-1}{v^{s a+1}}>0 \quad \text { as } u \rightarrow \infty .
$$

(3) Let $F$ be the Weibull distribution with tail

$$
\bar{F}(x)=\exp \left\{-\lambda x^{b}\right\}, \quad \lambda>0, \quad b \in(0,1), \quad x \geq 0 .
$$

We analogously obtain that, for any $v>1$ and $u>0$,

$$
D(u) \geq \frac{1}{v}\left[1-\frac{\bar{F}(v u)}{\bar{F}(u)}\right]=\frac{1-\exp \left\{-\lambda\left(v^{b}-1\right) u^{b}\right\}}{v} \rightarrow \frac{1}{v}>0 \quad \text { as } u \rightarrow \infty .
$$

Example 4.2. Next, we study the convergence rate of the deviation $\Gamma(u)$ to 0 as $u \rightarrow \infty$ :

(1) Clearly, under the assumption that (2.2) holds for some $v>1$, the inequality (2.4) implies

$$
\Gamma(u)=\mathrm{O}\left(u^{-1}+\bar{\Delta}(u)+\bar{F}(u)\right) .
$$

(2) Recently, Mikosch and Nagaev (2001) examined the case where the e.d.f. $F$ of claim size belongs to the class $\mathcal{D}$ of d.f.'s with dominatedly varying tails. We say a d.f. $F$ supported on $[0, \infty)$ belongs to the class $\mathcal{D}$ iff for some (or equivalently for any) $0<c<1$, it holds that

$$
\limsup _{x \rightarrow \infty} \frac{\bar{F}(c x)}{\bar{F}(x)}<\infty .
$$

The work in Mikosch and Nagaev (2001) indicates that if the e.d.f. $F \in \mathcal{D}$ with a finite mean, then $\Delta(u)=$ $\mathrm{O}\left(u^{-1}\right)$ and therefore $\bar{\Delta}(u)=\mathrm{O}\left(u^{-1}\right)$ as $u \rightarrow \infty$. From this and (4.3) we immediately obtain the following proposition.

Proposition 4.1. In the classical model with a constant interest force $r \geq 0$, if the e.d.f. $F$ of the claim size belongs to $\mathcal{A} \cap \mathcal{D}$ and has a finite mean, then $\Gamma(u)=\mathrm{O}\left(u^{-1}\right)$ as $u \rightarrow \infty$.

We remark that the intersection $\mathcal{A} \cap \mathcal{D}$ is a large subclass of heavy-tailed distributions. For example, if the e.d.f. $F \in \operatorname{ERV}(-\alpha,-\beta)$ for $1<\alpha \leq \beta<\infty$, i.e.

$$
v^{-\beta} \leq \liminf _{x \rightarrow \infty} \frac{\bar{F}(v x)}{\bar{F}(x)} \leq \limsup _{x \rightarrow \infty} \frac{\bar{F}(v x)}{\bar{F}(x)} \leq v^{-\alpha} \text { for any } v>1,
$$

then $F$ satisfies all the conditions in Proposition 4.1, see Bingham et al. (1987) and Tang et al. (2001) for details. Clearly, if $\alpha=\beta$ then the class $\operatorname{ERV}(-\alpha,-\beta)$ coincides with the class $\mathcal{R}_{-\alpha}$.

(3) We notice that in Proposition 4.1 the finite mean of the e.d.f. $F$ should be assumed in order for us to apply the related result in Mikosch and Nagaev (2001). So it excludes the case where $F$ follows a Pareto distribution with $0<a<1$ (recall (4.1)), which is of interests in insurance practice. In this case, from the relation (1.17) of Kalashnikov and Tsitsiashvili (2000) and the concrete value of $\beta_{F}$ given in p. 268 of that paper, we can obtain the asymptotic expression $\Delta(u)=\mathrm{O}\left(u^{-a /(a+1)}\right)$ as $u \rightarrow \infty$. This formula, in combination with (4.3), yields $\Gamma(u)=\mathrm{O}\left(u^{-a /(a+1)}\right)$ as $u \rightarrow \infty$. This convergence rate is slightly worse than that in Proposition 4.1. 
(4) If $F$ follows the Weibull distribution given in (4.2), then, by the same approach and using the value of $\beta_{F}$ given in p. 269 of Kalashnikov and Tsitsiashvili (2000), we obtain $\Delta(u)=\mathrm{O}\left((\ln u)^{1 / b} u^{b-1}\right)$ as $u \rightarrow \infty$. This formula, together with (4.3), yields $\Gamma(u)=\mathrm{O}\left((\ln u)^{1 / b} u^{b-1}\right)$ as $u \rightarrow \infty$.

(5) If $F$ follows the log-normal distribution with the density function:

$$
f(x)=\frac{1}{\sqrt{2 \pi} x} \exp \left\{-\frac{\ln ^{2} x}{2}\right\}, \quad x>0,
$$

then, the same approach, together with the value of $\beta_{F}$ given in p. 270 of Kalashnikov and Tsitsiashvili (2000), gives $\Delta(u)=\mathrm{O}\left(u^{-1}(\ln u) \exp \{\sqrt{2 \ln u}\}\right)$ as $u \rightarrow \infty$, from which we similarly obtain $\Gamma(u)=$ $\mathrm{O}\left(u^{-1}(\ln u) \exp \{\sqrt{2 \ln u}\}\right)$ as $u \rightarrow \infty$.

\section{Numerical results}

We write, for $u>0$,

$$
\begin{aligned}
& \psi_{r}^{-}(u)=\max \left\{\left[1+\Gamma_{-}(u)\right] \frac{\rho}{r u} D(u) \bar{F}(u), 0\right\}, \\
& \psi_{r}^{+}(u)=\left[1+\Gamma_{+}(u)\right] \frac{\rho}{r u} D(u) \bar{F}(u) \quad \text { and } \quad \psi_{r}^{*}(u)=\frac{\rho}{r u} D(u) \bar{F}(u) .
\end{aligned}
$$

Under the conditions of Theorem 2.1, we know from Corollary 2.1 that $\psi_{r}(u) \sim \psi_{r}^{*}(u)$ as $u \rightarrow \infty$; under the conditions of Theorem 2.2 we know that $\psi_{r}^{-}(u) \leq \psi_{r}(u) \leq \psi_{r}^{+}(u)$ for $u>0$. We proceed to the calculation of these estimates in the Pareto and Weibull cases. The upper bounds for the ruin probability $\psi_{0}(u)$ involved can be found in Kalashnikov and Tsitsiashvili (2000). We take the numerical results produced in the package Mathematica. In each table we vary the interest force from 0.01 to 0.31 by step 0.1 . For simplicity we assume $c=1$.

In the first case we assume the e.d.f. $F$ follows a Pareto distribution, the tail of which has form

$$
\bar{F}(u)=(1+b u)^{-a}, \quad a, b, u>0 .
$$

We take the parameters above with values $a=3, b=0.5$. In Tables $1-3$ we take the numerical results for $\rho=0.1$ and $u=9,100,1000$.

In Tables $4-6$ we take $\rho=0.9$ and $u=9,100,1000$.

Table 1

Pareto case with $a=3, b=0.5, \rho=0.1, u=9, \psi_{0}(u)=0.000725, \Delta(u)=0.0855969, D(u)=0.718924$

\begin{tabular}{llll}
\hline$r$ & $\psi_{r}^{*}(u)$ & $\psi_{r}^{-}(u)$ & $\psi_{r}^{+}(u)$ \\
\hline 0.01 & 0.00480123 & 0 & 0.00710027 \\
0.11 & 0.000436475 & 0.000116647 & 0.000574674 \\
0.21 & 0.00022863 & 0.000110868 & 0.000287031 \\
0.31 & 0.000154878 & 0.0000928057 & 0.000189465
\end{tabular}

Table 2

Pareto case with $a=3, b=0.5, \rho=0.1, u=100, \psi_{0}(u)=8.44 \times 10^{-7}, \Delta(u)=0.007617, D(u)=0.74702$

\begin{tabular}{llll}
\hline$r$ & $\psi_{r}^{*}(u)$ & $\psi_{r}^{-}(u)$ & $\psi_{r}^{+}(u)$ \\
\hline 0.01 & $5.63147 \times 10^{-7}$ & $1.84765 \times 10^{-7}$ & $6.64245 \times 10^{-7}$ \\
0.11 & $5.11952 \times 10^{-8}$ & $4.5352 \times 10^{-8}$ & $5.3162 \times 10^{-8}$ \\
0.21 & $2.68165 \times 10^{-8}$ & $2.51156 \times 10^{-8}$ & $2.75028 \times 10^{-8}$ \\
0.31 & $1.8166 \times 10^{-8}$ & $1.73592 \times 10^{-8}$ & $1.85435 \times 10^{-8}$ \\
\hline
\end{tabular}


Table 3

Pareto case with $a=3, b=0.5, \rho=0.1, u=1000, \psi_{0}(u)=8.85 \times 10^{-10}, \Delta(u)=0.00161071, D(u)=0.749592$

\begin{tabular}{lllr}
\hline$r$ & $\psi_{r}^{*}(u)$ & $\psi_{r}^{-}(u)$ & $\psi_{r}^{+}(u)$ \\
\hline 0.01 & $5.9609 \times 10^{-11}$ & $5.23476 \times 10^{-11}$ & $6.15473 \times 10^{-11}$ \\
0.11 & $5.419 \times 10^{-12}$ & $5.35095 \times 10^{-12}$ & $5.44695 \times 10^{-12}$ \\
0.21 & $2.83852 \times 10^{-12}$ & $2.81905 \times 10^{-12}$ & $2.84911 \times 10^{-12}$ \\
0.31 & $1.92287 \times 10^{-12}$ & $1.91359 \times 10^{-12}$ & $1.92907 \times 10^{-12}$
\end{tabular}

Table 4

Pareto-like case with $a=3, b=0.5, \rho=0.9, u=9, \psi_{0}(u)=0.356, \Delta(u)=5.58106, D(u)=0.718924$

\begin{tabular}{llll}
\hline$r$ & $\psi_{r}^{*}(u)$ & $\psi_{r}^{-}(u)$ & $\psi_{r}^{+}(u)$ \\
\hline 0.01 & 0.0432111 & 0 & 0.612822 \\
0.11 & 0.00392828 & 0 & 0.0550738 \\
0.21 & 0.00205767 & 0 & 0.0287223 \\
0.31 & 0.0013939 & 0 & 0.0194123 \\
\hline
\end{tabular}

Table 5

Pareto case with $a=3, b=0.5, \rho=0.9, u=100, \psi_{0}(u)=0.000348, \Delta(u)=4.12917, D(u)=0.74702$

\begin{tabular}{llll}
\hline$r$ & $\psi_{r}^{*}(u)$ & $\psi_{r}^{-}(u)$ & $\psi_{r}^{+}(u)$ \\
\hline 0.01 & $5.06832 \times 10^{-6}$ & 0 & 0.0000339539 \\
0.11 & $4.60756 \times 10^{-7}$ & 0 & $3.0217 \times 10^{-6}$ \\
0.21 & $2.41349 \times 10^{-7}$ & 0 & $1.5797 \times 10^{-6}$ \\
0.31 & $1.63494 \times 10^{-7}$ & 0 & $1.06933 \times 10^{-6}$ \\
\hline
\end{tabular}

Table 6

Pareto case with $a=3, b=0.5, \rho=0.9, u=1000, \psi_{0}(u)=8.24 \times 10^{-8}, \Delta(u)=0.151325, D(u)=0.749592$

\begin{tabular}{llll}
\hline$r$ & $\psi_{r}^{*}(u)$ & $\psi_{r}^{-}(u)$ & $\psi_{r}^{+}(u)$ \\
\hline 0.01 & $5.36481 \times 10^{-10}$ & $4.44297 \times 10^{-10}$ & $6.61076 \times 10^{-10}$ \\
0.11 & $4.8771 \times 10^{-11}$ & $4.57194 \times 10^{-11}$ & $5.87635 \times 10^{-11}$ \\
0.21 & $2.55467 \times 10^{-11}$ & $2.40937 \times 10^{-11}$ & $3.07444 \times 10^{-11}$ \\
0.31 & $1.73058 \times 10^{-11}$ & $1.63568 \times 10^{-11}$ & $2.08181 \times 10^{-11}$ \\
\hline
\end{tabular}

Table 7

Pareto case with $a=5, b=0.25, \rho=0.1, u=9, \psi_{0}(u)=0.000355, \Delta(u)=0.158478, D(u)=0.78556$

\begin{tabular}{llll}
\hline$r$ & $\psi_{r}^{*}(u)$ & $\psi_{r}^{-}(u)$ & $\psi_{r}^{+}(u)$ \\
\hline 0.01 & 0.00240724 & 0 & 0.003497 \\
0.11 & 0.00021884 & 0.0000693594 & 0.000293123 \\
0.21 & 0.000114631 & 0.0000591667 & 0.000148644 \\
0.31 & 0.0000776531 & 0.0000482031 & 0.0000989523 \\
\hline
\end{tabular}

Now we assume $a=5, b=0.25$. In Tables 7-9 we take $\rho=0.1$ and $u=9,100,1000$.

In Tables 10-12 we take $\rho=0.9$ and $u=9,100,1000$.

In the second case we assume the e.d.f. $F$ follows a Weibull distribution with the form (4.2). We take the parameters in (4.2) with values $\lambda=4.52874, b=0.1$.

In Tables 13-16 we take $\rho=0.5$ and $u=10,100,1000,10000$.

In Tables 17-20 we take $\rho=0.95$ and $u=10,100,1000,10,000$. 
Table 8

Pareto case with $a=5, b=0.25, \rho=0.1, u=100, \psi_{0}(u)=9.47 \times 10^{-9}, \Delta(u)=0.0126497, D(u)=0.828618$

\begin{tabular}{llll}
\hline$r$ & $\psi_{r}^{*}(u)$ & $\psi_{r}^{-}(u)$ & $\psi_{r}^{+}(u)$ \\
\hline 0.01 & $6.97409 \times 10^{-9}$ & $2.74757 \times 10^{-9}$ & $7.80178 \times 10^{-9}$ \\
0.11 & $6.34008 \times 10^{-10}$ & $5.68588 \times 10^{-10}$ & $6.54614 \times 10^{-10}$ \\
0.21 & $3.32099 \times 10^{-10}$ & $3.13013 \times 10^{-10}$ & $3.40291 \times 10^{-10}$ \\
0.31 & $2.24971 \times 10^{-10}$ & $2.15898 \times 10^{-10}$ & $2.29859 \times 10^{-10}$ \\
\hline
\end{tabular}

Table 9

Pareto case with $a=5, b=0.25, \rho=0.1, u=1000, \psi_{0}(u)=1.12 \times 10^{-13}, \Delta(u)=0.00422063, D(u)=1$

\begin{tabular}{llll}
\hline$r$ & $\psi_{r}^{*}(u)$ & $\psi_{r}^{-}(u)$ & $\psi_{r}^{+}(u)$ \\
\hline 0.01 & $1.00376 \times 10^{-14}$ & $9.12512 \times 10^{-15}$ & $1.008 \times 10^{-14}$ \\
0.11 & $9.12512 \times 10^{-16}$ & $9.04291 \times 10^{-16}$ & $9.16364 \times 10^{-16}$ \\
0.21 & $4.77983 \times 10^{-16}$ & $4.75717 \times 10^{-16}$ & $4.8 \times 10^{-16}$ \\
0.31 & $3.23795 \times 10^{-16}$ & $3.22754 \times 10^{-16}$ & $3.25161 \times 10^{-16}$ \\
\hline
\end{tabular}

Table 10

Pareto case with $a=5, b=0.25, \rho=0.9, u=9, \psi_{0}(u)=0.364, \Delta(u)=13.6648, D(u)=0.78556$

\begin{tabular}{llll}
\hline$r$ & $\psi_{r}^{*}(u)$ & $\psi_{r}^{-}(u)$ & $\psi_{r}^{+}(u)$ \\
\hline 0.01 & 0.0216652 & 0 & 0.635431 \\
0.11 & 0.00196956 & 0 & 0.0575433 \\
0.21 & 0.00103168 & 0 & 0.0300977 \\
0.31 & 0.000698878 & 0 & 0.0203731 \\
\hline
\end{tabular}

Table 11

Pareto case with $a=5, b=0.25, \rho=0.9, u=100, \psi_{0}(u)=0.00013, \Delta(u)=170.62, D(u)=0.828618$

\begin{tabular}{llll}
\hline$r$ & $\psi_{r}^{*}(u)$ & $\psi_{r}^{-}(u)$ & $\psi_{r}^{+}(u)$ \\
\hline 0.01 & $6.27668 \times 10^{-8}$ & 0 & 0.0000129952 \\
0.11 & $5.70607 \times 10^{-8}$ & 0 & $1.18089 \times 10^{-6}$ \\
0.21 & $2.98889 \times 10^{-8}$ & 0 & $6.18538 \times 10^{-7}$ \\
0.31 & $2.02474 \times 10^{-8}$ & 0 & $4.19004 \times 10^{-7}$ \\
\hline
\end{tabular}

Table 12

Pareto case with $a=5, b=0.25, \rho=0.9, u=1000, \psi_{0}(u)=1.14 \times 10^{-11}, \Delta(u)=0.261917, D(u)=1$

\begin{tabular}{llll}
\hline$r$ & $\psi_{r}^{*}(u)$ & $\psi_{r}^{-}(u)$ & $\psi_{r}^{+}(u)$ \\
\hline 0.01 & $9.03387 \times 10^{-14}$ & $8.21261 \times 10^{-14}$ & $1.14 \times 10^{-13}$ \\
0.11 & $8.21261 \times 10^{-15}$ & $8.13862 \times 10^{-15}$ & $1.03636 \times 10^{-14}$ \\
0.21 & $4.30184 \times 10^{-15}$ & $4.28146 \times 10^{-15}$ & $5.42857 \times 10^{-15}$ \\
0.31 & $2.91415 \times 10^{-15}$ & $2.90478 \times 10^{-15}$ & $3.67742 \times 10^{-15}$ \\
\hline
\end{tabular}

Table 13

Weibull case with $\lambda=4.52874, b=0.1, \rho=0.5, u=10, \psi_{0}(u)=0.00335, \Delta(u)=0.00255419, D(u)=0.377721$

\begin{tabular}{llll}
\hline$r$ & $\psi_{r}^{*}(u)$ & $\psi_{r}^{-}(u)$ & $\psi_{r}^{+}(u)$ \\
\hline 0.01 & 0.00631071 & 0 & 0.0158612 \\
0.11 & 0.000573701 & 0 & 0.00103277 \\
0.21 & 0.00030051 & 0.000040936 & 0.000464925 \\
0.31 & 0.000203571 & 0.0000701342 & 0.000288563 \\
\hline
\end{tabular}


Table 14

Weibull case with $\lambda=4.52874, b=0.1, \rho=0.5, u=100, \psi_{0}(u)=0.000766, \Delta(u)=0.00324607, D(u)=0.431774$

\begin{tabular}{llll}
\hline$r$ & $\psi_{r}^{*}(u)$ & $\psi_{r}^{-}(u)$ & $\psi_{r}^{+}(u)$ \\
\hline 0.01 & 0.000164834 & 0 & 0.00027483 \\
0.11 & 0.0000149849 & 0.0000120136 & 0.0000167677 \\
0.21 & $7.84925 \times 10^{-6}$ & $6.98146 \times 10^{-6}$ & $8.39178 \times 10^{-6}$ \\
0.31 & $5.31724 \times 10^{-6}$ & $4.9043 \times 10^{-6}$ & $5.58536 \times 10^{-6}$ \\
\hline
\end{tabular}

Table 15

Weibull case with $\lambda=4.52874, b=0.1, \rho=0.5, u=1000, \psi_{0}(u)=0.00012, \Delta(u)=0.00803757, D(u)=0.487477$

\begin{tabular}{llll}
\hline$r$ & $\psi_{r}^{*}(u)$ & $\psi_{r}^{-}(u)$ & $\psi_{r}^{+}(u)$ \\
\hline 0.01 & $2.90154 \times 10^{-6}$ & $2.33555 \times 10^{-6}$ & $3.22743 \times 10^{-6}$ \\
0.11 & $2.63777 \times 10^{-7}$ & $2.56639 \times 10^{-7}$ & $2.7069 \times 10^{-7}$ \\
0.21 & $1.38169 \times 10^{-7}$ & $1.3564 \times 10^{-7}$ & $1.4117 \times 10^{-7}$ \\
0.31 & $9.35981 \times 10^{-8}$ & $9.21779 \times 10^{-8}$ & $9.5481 \times 10^{-8}$
\end{tabular}

Table 16

Weibull case with $\lambda=4.52874, b=0.1, \rho=0.5, u=10000, \psi_{0}(u)=0.0000115, \Delta(u)=0.00252021, D(u)=0.543501$

\begin{tabular}{llll}
\hline$r$ & $\psi_{r}^{*}(u)$ & $\psi_{r}^{-}(u)$ & $\psi_{r}^{+}(u)$ \\
\hline 0.01 & $3.11727 \times 10^{-8}$ & $3.05386 \times 10^{-8}$ & $3.15772 \times 10^{-8}$ \\
0.11 & $2.83388 \times 10^{-9}$ & $2.82312 \times 10^{-9}$ & $2.84925 \times 10^{-9}$ \\
0.21 & $1.48442 \times 10^{-9}$ & $1.47996 \times 10^{-9}$ & $1.49192 \times 10^{-9}$ \\
0.31 & $1.00557 \times 10^{-9}$ & $1.00284 \times 10^{-9}$ & $1.01053 \times 10^{-9}$ \\
\hline
\end{tabular}

Table 17

Weibull case with $\lambda=4.52874, b=0.1, \rho=0.95, u=10, \psi_{0}(u)=0.0658, \Delta(u)=0.0364189, D(u)=0.377721$

\begin{tabular}{llll}
\hline$r$ & $\psi_{r}^{*}(u)$ & $\psi_{r}^{-}(u)$ & $\psi_{r}^{+}(u)$ \\
\hline 0.01 & 0.0119903 & 0 & 0.0334215 \\
0.11 & 0.00109003 & 0 & 0.00226093 \\
0.21 & 0.000570969 & 0 & 0.0010398 \\
0.31 & 0.000386785 & 0.0000673069 & 0.000654247 \\
\hline
\end{tabular}

Table 18

Weibull case with $\lambda=4.52874, b=0.1, \rho=0.95, u=100, \psi_{0}(u)=0.0156, \Delta(u)=0.0753496, D(u)=0.431774$

\begin{tabular}{llll}
\hline$r$ & $\psi_{r}^{*}(u)$ & $\psi_{r}^{-}(u)$ & $\psi_{r}^{+}(u)$ \\
\hline 0.01 & 0.000313185 & 0 & 0.000586281 \\
0.11 & 0.0000284714 & 0.0000195145 & 0.0000376861 \\
0.21 & 0.0000149136 & 0.0000115302 & 0.0000189969 \\
0.31 & 0.0000101028 & 0.00000814317 & 0.00001268 \\
\hline
\end{tabular}

Table 19

Weibull case with $\lambda=4.52874, b=0.1, \rho=0.95, u=1000, \psi_{0}(u)=0.00246, \Delta(u)=0.0876195, D(u)=0.487477$

\begin{tabular}{lllr}
\hline$r$ & $\psi_{r}^{*}(u)$ & $\psi_{r}^{-}(u)$ & $\psi_{r}^{+}(u)$ \\
\hline 0.01 & $5.51293 \times 10^{-6}$ & $3.96143 \times 10^{-6}$ & $7.06109 \times 10^{-6}$ \\
0.11 & $5.01175 \times 10^{-7}$ & $4.44331 \times 10^{-7}$ & $5.98762 \times 10^{-7}$ \\
0.21 & $2.6252 \times 10^{-7}$ & $2.35044 \times 10^{-7}$ & $3.12459 \times 10^{-7}$ \\
0.31 & $1.77836 \times 10^{-7}$ & $1.59779 \times 10^{-7}$ & $2.11381 \times 10^{-7}$ \\
\hline
\end{tabular}


Table 20

Weibull case with $\lambda=4.52874, b=0.1, \rho=0.95, u=10000, \psi_{0}(u)=0.00024, \Delta(u)=0.101166, D(u)=0.543501$

\begin{tabular}{llll}
\hline$r$ & $\psi_{r}^{*}(u)$ & $\psi_{r}^{-}(u)$ & $\psi_{r}^{+}(u)$ \\
\hline 0.01 & $5.92282 \times 10^{-8}$ & $5.31033 \times 10^{-8}$ & $7.07742 \times 10^{-8}$ \\
0.11 & $5.38438 \times 10^{-9}$ & $4.91666 \times 10^{-9}$ & $6.39334 \times 10^{-9}$ \\
0.21 & $2.82039 \times 10^{-9}$ & $2.57764 \times 10^{-9}$ & $3.34787 \times 10^{-9}$ \\
0.31 & $1.91059 \times 10^{-9}$ & $1.74668 \times 10^{-9}$ & $2.26767 \times 10^{-9}$ \\
\hline
\end{tabular}

\section{Acknowledgements}

We feel the duty to express our deep gratitude to the late Prof. V.V. Kalashnikov for his numerous valuable discussions on this paper. We would like to thank the anonymous referee for the constructive suggestions and comments on the previous versions of this paper.

\section{References}

Asmussen, S., 1998. Subexponential asymptotics for stochastic processes: extremal behaviour, stationary distributions and first passage probabilities. The Annals of Applied Probability 8, 354-374.

Asmussen, S., 2000. Ruin Probabilities. World Scientific, Singapore.

Asmussen, S., Kalashnikov, V., Klüppelberg, C., Konstantinides, D., Tsitsiashvili, G.Sh., 2002. A local limit theorem for random walk maxima with heavy-tails. Statistics and Probability Letters 56, 399-404.

Bingham, N.H., Goldie, C.M., Teugels, J.L., 1987. Regular Variation. Cambridge University Press, Cambridge.

Embrechts, P., Veraverbeke, N., 1982. Estimates for the probability of ruin with special emphasis on the possibility of large claims. Insurance: Mathematics and Economics 1, 55-72.

Embrechts, P., Klüppelberg, C., Mikosch, T., 1997. Modelling Extremal Events for Insurance and Finance. Springer, New York.

Feller, W., 1971. An Introduction to Probability Theory and its Applications, vol. II. Wiley, New York.

Kalashnikov, V.V., Konstantinides, D., 2000. Ruin under interest force and subexponential claims: a simple treatment. Insurance: Mathematics and Economics 27, 145-149.

Kalashnikov, V.V., Tsitsiashvili, G.Sh., 1999. Tails of waiting times and their bounds. Queueing Systems 32, $257-283$.

Kalashnikov, V.V., Tsitsiashvili, G.Sh., 2000. Tight approximation of basic characteristics of classical and non-classical surplus process. ARCH00V210(2000-9), vol. 2, pp. 251-293.

Klüppelberg, C., Stadtmüller, U., 1998. Ruin probabilities in the presence of heavy-tails and interest rates. Scandinavian Actuarial Journal, $49-58$.

Mikosch, T., Nagaev, A., 2001. Rates in approximations to ruin probabilities for heavy-tailed distributions. Extremes 4 (1), 67-78.

Rolski, T., Schmidli, H., Schmidt, V., Teugels, J., 1999. Stochastic Processes for Insurance and Finance. Wiley, New York.

Sundt, B., Teugels, J.L., 1995. Ruin estimates under interest force. Insurance: Mathematics and Economics 16, 7-22.

Sundt, B., Teugels, J.L., 1997. The adjustment function in ruin estimates under interest force. Insurance: Mathematics and Economics 19, 85-94.

Tang, Q.H., Su, C., Jiang, T., Zhang, J.S., 2001. Large deviations for heavy-tailed random sums in compound renewal model. Statistics and Probability Letters 52, 91-100. 